\title{
EOG Pattern Recognition Trial for a Human Computer Interface
}

\author{
Sara Brunner ${ }^{1}$, Sten Hanke ${ }^{2}$, Siegfried Wassertheuer ${ }^{2}$, and Andreas Hochgatterer ${ }^{2}$ \\ ${ }^{1}$ University of Applied Sciences Linz - FH Linz Medizintechnik \\ ${ }^{2}$ Austrian Research Centers GmbH - ARC \\ sten.hankedarcsmed.at
}

\begin{abstract}
The setup of a human computer interaction electrooculography (EOG) measurement trail for developing pattern recognition algorithms is described. With an easy to wear EOG measurement device we relized performance tests with a group of normal individuals as well as with one individual suffering from multiple sclerosis (MS). The individuals had to perform different eye movement patterns for coding information to control the environment. Different patterns of recognition in the time domain have been tried and implemented to perform online performance tests. The aim is to develop an EOG based communication device passed on pattern recognition algorithms for user with limited functionality.
\end{abstract}

Keywords: Electrooculography, EOG, pattern recognition, human computer interface.

\section{Introduction}

The bulk of human computer interactions occur via standard input devices like keyboard or mouse. An EOG-input device could enable handicapped people who are not able to use standard input devices, to use the computer $[4,5]$.

We developed a new possibility to apply electrodes in an effective, but simple way to the users face. Due to the trigger generation via pattern recognition the device will be usable even from persons suffering from amyotrophic lateral sclerosis (ALS) or multiple sclerosis (MS) [4,5].

To obtain a stable human machine interface that is independent of the current eye position, we investigated if eye movement patterns can be used to encode distinct commands.

We studied if EOG-signals, measured by the EOG-head device could provide sufficient signal-to-noise ratio for subsequent signal processing, even if no conductive paste is used and the skin is not pretreated.

Three different approaches of pattern recognition in time-domain has been developed, tested and evaluated. Finally, an online pattern recognition was realized in Matlab. 
Patients suffering from ALS, high cervical spinal cord injury, or advanced cases of MS are highly constricted in their motility and communication, and are therefore excluded from interacting with computers and communication systems. Our system provides a comfortable solution for communication with eye movements even if the eye movements are already influenced by any kind of disease [1].

During the study there appeared several questions concerning performance and feasibility for recapitulating the different patterns. One of these questions was the usability for ALS or MS patients and their efficiency for generating and repeating different eye movement patterns.

We also wanted to have a look at the coherence between the generated eye movement and the submitted information. The study was aimed to clarify what kind of eye movement patterns will be generated and how easily they can be distinguished using different appendages of pattern recognition algorithms.

\section{Material and Methods}

So far six healthy subjects and one MS-patient have been tested. The MS-patient is able to move his head and to speak slowly but cannot move his limbs. Presently he uses a head-mounted mouse for computer control. Table 1 gives information on the different subjects involved in the experiments. The head circumference was important, due to a different head circumference there will be a different positioning and pressure of the measurement electrodes because of the fixed head device (Figure 1). This could cause a different magnitude of the EOG signals which should, as a demand, have no influence on the pattern recognition. The head circumference was measured above the eyebrows and above the ears, and was used only for orientation.

Table 1. Participants of the experiment ranged by age

\begin{tabular}{llllll}
\hline & Sex & Age & $\begin{array}{l}\text { Head } \\
\text { Circumference } \\
{[\mathbf{c m}]}\end{array}$ & Diseases & $\begin{array}{l}\text { Glasses/ } \\
\text { Lenses }\end{array}$ \\
\hline Subject 5 & male & 17 & 56.0 & No & No \\
Subject 1 & female & 18 & 53.0 & No & Yes \\
Subject 3 & male & 20 & 58.0 & No & No \\
Subject 7 & female & 22 & 56.0 & No & Yes \\
Subject 2 & male & 27 & 58.0 & No & Yes \\
Subject 4 & female & 29 & 53.0 & No & No \\
Subject 6 & male & 41 & 54.5 & MS & Yes \\
\hline
\end{tabular}

For getting a huge number of different eye movement sequences, the subjects had to code 18 different terms, divided into four groups. Each pattern was recorded three times per session, and two sessions were held within a month. The length of recorded data was limited to three seconds (600 data points).

The first group was the 'direction' block: the subjects had to code 'left', 'right', 'up', 'down' and 'enter'. The second block was a 'number' block, where numbers 
from one to five had to be converted into eye movements. The third block contained the commands 'yes', 'no', 'help' and 'switch light' and the fourth block was the 'color' block, containing 'red', 'blue', 'yellow' and 'green'. The subjects could choose freely how to code the terms, but were not allowed to use the same eye movement sequence twice in one block. The subjects recorded their eye movement sequences in order to remind them at the second session, when they had to redo the same movements for each command.

For a convenient handling of the EOG electrodes we developed a comfortable, simple head device which allows easy application of five gold cup electrodes to the subjects face without abrading the skin or using conductive paste. The electrodes were placed at the two outer canthi (horizontal EOG), above and under the right orbit (vertical EOG) and at the forehead (common electrode).
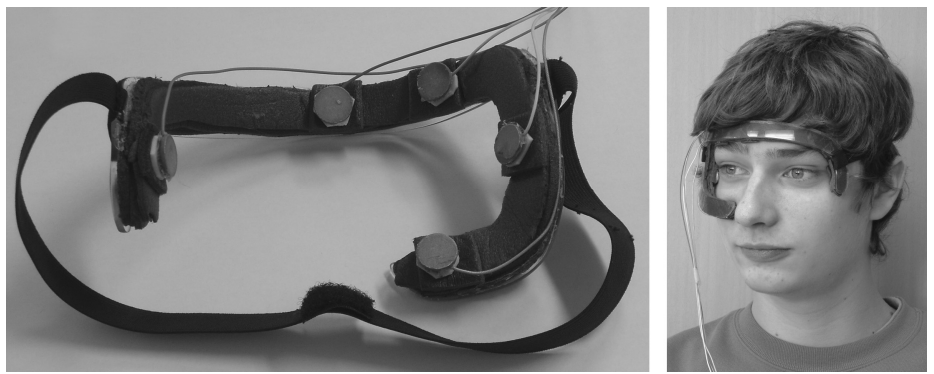

Fig. 1. Left: Five gold cup electrodes are integrated into the prototype of the EOG-Head Device, which is ergonomically designed for human heads. Right: A subject wearing the Head Device.

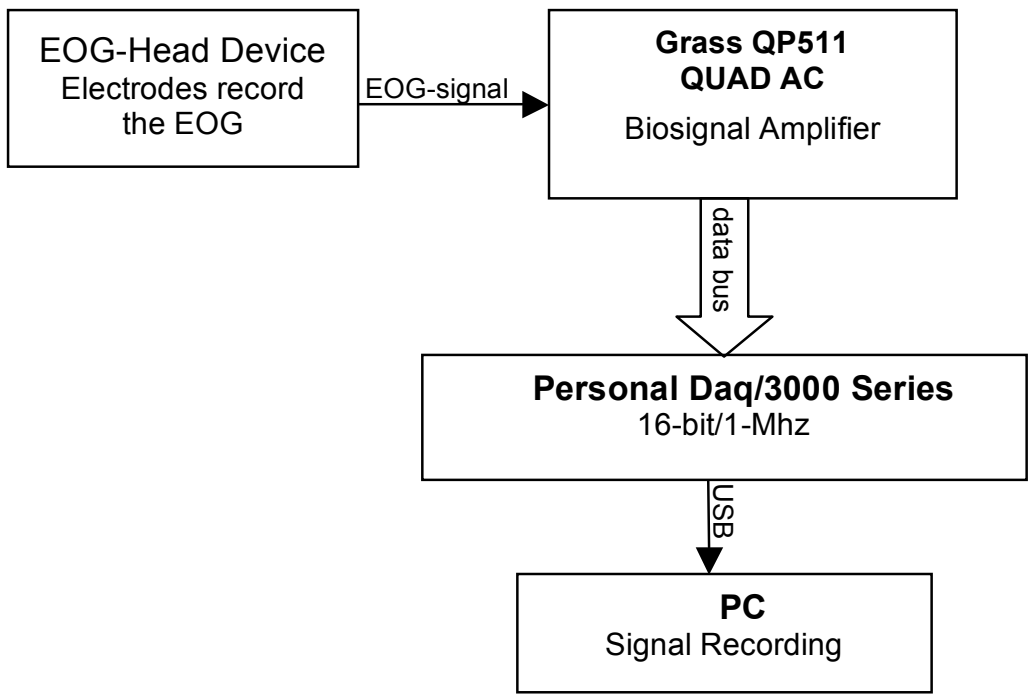

Fig. 2. Structure of the measurement chain $[4,5]$ 
For the horizontal and vertical measuring channel the two corresponding electrodes and the common electrode of the head device were connected respectively with the Grass QP511 Quad Amplifier System via a 3-lead input cable (CAB-21572). The electrode signals were amplified (factor 5000) and bandpass filtered $(1 \mathrm{~Hz}-300$ $\mathrm{Hz}$ [2], before they were sampled with the 16-bit/1-MHz analog-to-digital converter iotech Personal Daq/3000 Series. The sample rate was defined with $200 \mathrm{~Hz}$. The digital data were imported with Matlab - Data Acquisition Toolbox.

The first step in our analysis was to investigate the repeatability of the individual eye movement patterns, which was rated by the similarity of the repeated patterns. Then we evaluated three different procedures to classify the eye movement patterns:

1) The first method was to evaluate the correlation coefficient of reference patterns (one for each term) and the actual signal. All signals for correlation were 600 data points long. Signals were counted as 'equal' when the correlation coefficient was bigger than 0.6 with a confidence level of 90 percent.

$$
r=\frac{\sum_{i=1}^{n} x_{i} y_{i}-n \bar{x} \bar{y}}{\sqrt{\left(\sum_{i=1}^{n} x_{i}^{2}-n \bar{x}^{2}\right)\left(\sum_{i=1}^{n} y_{i}^{2}-n \bar{y}^{2}\right)}}, \quad-1 \leq r \leq 1
$$

Formula for the calculation of the correlation coefficient. [3].

2) From three recorded signals of one coded term an 'envelope' was built with tolerance ( 20 percent in amplitude, 10 data points in time). The aim was to get out of three generated signals, a signal course where all of the three generated patterns are fitting in. If incoming signals were enclosed by the 'envelope', a correlation of the eye movement sequence was supposed.

Especially for this method there was the problem to get an uniform starting point for each pattern. For the creation of the combined envelope it would be important to have all there pattern signals started at the same point in time to get the envelope not too fuzzy. But the question was if it is possible for the user to repeat the same pattern three times in serial with such an exact timing.

Therefore, would it be more difficult for the ALS and MS patients?

In our setup we recorded the different signal patterns after a light was appearing. In that case the user was noticed that recording started and got the instruction to perform the pattern of eye movement. The recording lasted for a defined period of time (3 seconds). The signal conditioning and mainly the calculation of the envelope was performed based on this recorded signal patterns.

Another approach would be to start the 3 seconds recording session when the software will detect the beginning of the pattern generated by the user. We discarded that method because it doesn't represents the different timings of the user when generating eye movement patterns, and so this different timings wouldn't be represented in a fuzzier envelope.

3) For the third procedure, five levels were defined and each band got a corresponding value. The levels have been defined in that way that they are uniformly distributed 
over the signal amplitude. For the horizontal EOG the levels were at 0, 0.2, 0.4, 0.6 and $0.8 \mathrm{mV}$; for the vertical EOG the levels were at $0,0.1,0.2,0.3$ and $0.4 \mathrm{mV}$ with corresponding values $0,0.25,0.5,0.75$ and 1 . An algorithm converted the signal into a sequence of values, which was then used to classify the pattern.

For the implementation of the online-pattern recognition we decided to use the correlation coefficient within the algorithm. The measurement chain was the same as described above, only the Matlab-script was changed so the signals were processed online. For pattern recognition, the subject repeated one eye movement sequence three times. The correlation coefficients of each pattern with each other pattern were computed and the sum of the two coefficients for one signal was calculated. The signal with the highest value correlated best with both other signals and it was thought that this signal would even be the best reference pattern for the following signals. The best data set got normalized and saved as reference pattern. Continually 600 values of the incoming EOG-signal were normalized and compared with the reference pattern. If the correlation coefficient was greater than 0.6 , the algorithm recognized the reference pattern.

The online-algorithm was tested with two subjects. They made eye-movementsequences built with less than three gazes changes and repeated them. For testing false-positive results the subjects were asked to look around the room or to read some text at the computer monitor.

\section{Results}

Our tests indicated that simple patterns can be better reproduced than complex ones: the data show an inverse relationship between the complexity of the pattern and the

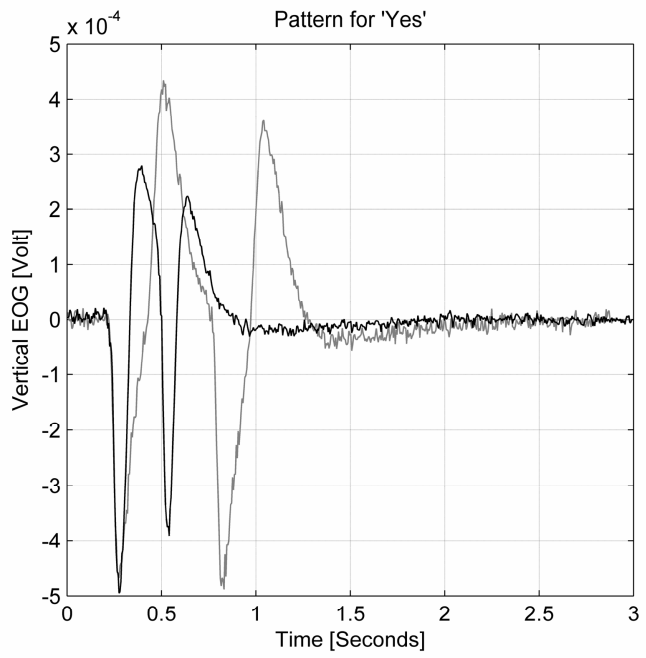

Fig. 3. For coding 'Yes' the subject made two blinks. The graph shows two out of six data sets. The correlation between these two signals is as low as 0.14 , although the subject believed it repeated the movements in the same way. 
probability of a correct reproduction. The best pattern reached a correlation coefficient between 0.93 and 0.99 for all six repeats, while for some complex patterns it was as low as 0.07 . We were surprised to find that vertical movements can be repeated more easily than horizontal ones, even if the horizontal ones have greater amplitudes.

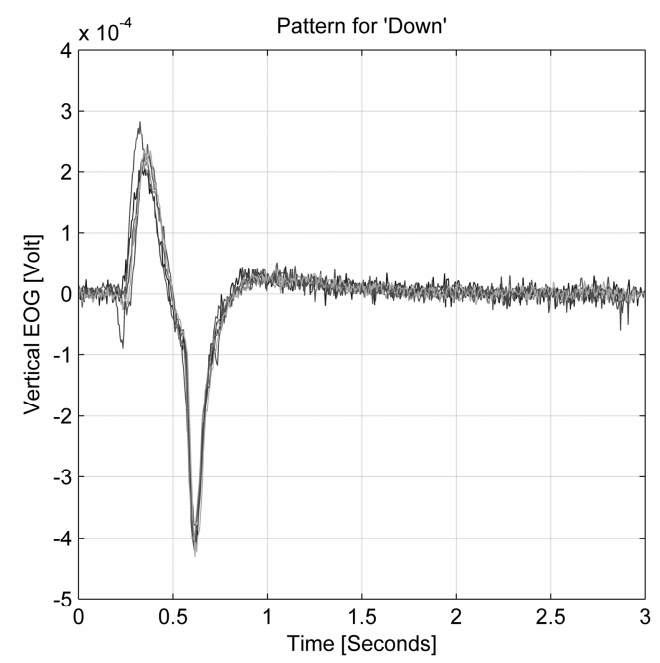

Fig. 4. The subject coded the term 'Down' by moving its eyes down and back to the middle position. The figure shows all six data sets which are highly congruent.

The relation between the term and the eye movement sequence showed that different subjects used the same sequences for coding terms. Especially in the 'direction' block every subject included the direction information in his/her eye movements. The subjects coded the numbers by counting either with blinks or with changes between up and down eye movements, e.g. three blinks or up-down-upmovement for coding the number three.

Interesting in that context was the exploration of the subjects to generated patterns. Without any specifications the subjects had the freedom to decide which kind of information (direction, color, etc.) they would code with what kind of eye movement. We had the assumption that there may be coherence. As mentioned, the coherence was apparent in the direction block but furthermore there was no appreciable relation. The terms within the blocks 'communication' and 'colors' were coded differently by every subject.

But the first trails with the MS patient showed that there maybe differences. The MS-patient built an exception: He coded every number by its own without any obvious context between them.

The best classification results were achieved with the correlation approach. Due to normalized data the influence of time delay within the eye movement sequences on the correlation coefficient was minimized. We got good results even with the 
envelope approach, but the algorithm could not compute an appropriate 'envelope' for every signal. As the third method, conversion between signal and assigned value, was too strict an identification of the pattern was hardly ever possible.

The online pattern recognition led to a probability of pattern recognition of 80 percent. The false-positive results were less than 5 percent. As the system was tested only with one pattern each session, false identified patterns were not obtained. The probability of correct assignment could be enhanced up to $95 \%$ by using simple, easy repeatable eye-movement-sequences.

\section{Discussion}

The EOG-Head Device is a useful, easy to handle tool for applying electrodes to the face. It allows EOG-recording with minimal preparatory work and required space, which is an advantage over already available gaze-tracking-systems or EOG-based control systems.

Due to the similar frequencies of different eye movements we could not achieve satisfactory results with pattern recognition in frequency domain. We analyzed the frequency spectrum of the eye movements 'left-right-middle' versus 'right-leftmiddle' and 'left-middle' versus 'right-middle'. The frequencies were all in the range of 0 to $15 \mathrm{~Hz}$ and a significant difference between them was not observable.

Our tests indicated that for a successful transfer of this technology from the laboratory to the bedside, certain minimum criteria have to be met. For example, the patients must be able to repeat the pattern often enough to train the system with sufficient ease. This is not a restriction for healthy subjects, and the technology holds the promise of a flexible, gaze independent human machine interface.

It has to be investigated if eye movements can be trained in order to improve the correlation between EOG-signals derived from subjectively equal eye movements. Therefore, it will be important to make the pattern recognition as fuzzy as essential but give the chance to distinguish between similar patterns. The similarity of the patterns depends on the possibility to repeat and replicate the single pattern. Therefore, in the following studies it should be clarified which patterns could be reproduced and are still to be distinguished in automatic detection. Furthermore it must be clarified if for MS and ALS patients some patterns are more difficult to replicate like it seems according to the pilot survey.

A possible experiment design could have five default eye movement sequences with three gaze changes each. The subjects will have to train these movements with a feedback mechanism. At first the goal could be to repeat the pattern three times, so that the computer can accept them as 'equal'. Advanced subject will have to repeat the same eye movements within a longer period of time (e.g. every 15 minutes) to see if people are able to train their eye movements.

While the data are currently evaluated in MATLAB, the final online pattern recognition algorithm will be implemented in a microcontroller MSP430 connected to a wireless transmission unit. 


\section{References}

1. Jacobs, L., Bozian, D., Heffner Jr., RR., Barron, SA.: An eye movement disorder in amytrophic lateral sclerosis. Neurology 31, 1282-1287 (1981)

2. Oster, PJ., Stern, JA.: Electrooculography. In: Martin, I., Venables, PH. (eds.) Techniques in Psychophysiology, John Wiley \& Sons, New York (1980)

3. Papula, L.: Mathematische Formelsammlung für Ingenieure und Naturwissenschaftler, 8. Auflage, Vieweg \& Sohn Verlag Wiesbaden (2003)

4. Hanke, S., Mina, S., Morgenstern, U., Bammer, M., Poll, R.: Utilization of an EOG based controlling device with Body Area Network connectivity. In: proceedings DGBMT 2005 Nuernberg Germany (2005)

5. Hanke, S., Mina, S.: EOG controlled computer system with Body Area Network connectivity. In: proceedings OEGBMT 2004 Graz Austria (2004) 